\title{
The Fate of Russian Translations of Cantor
}

\section{by Galina Sinkevich*}

\begin{abstract}
This is the history of translating Cantor's works into Russian from 1892 to 1985 in Odessa, Moscow, Tomsk, Kazan, S.-Petersburg, Leningrad. Mathematicians and philosophers in Russia took the ideas of the theory of sets enthusiastically. Such renowned scholars and scientists as Timchenko, Shatunovsky, Vasiliev, Florensky, Mlodzeevsky, Nekrasov, Zhegalkin, Yushkevich Sr., Fet, Yushkevich Jr., Kolmogorov, and Medvedev took part in their popularisation. In 1970 Academician Pontryagin rated the theory of sets as useless for young mathematicians, and the translated works of Cantor were not published. This article first describes the tragic fate of this translation.
\end{abstract}

MSC 2010 subject classifications: 01A60, 01A70, $01 \mathrm{~A} 85$.

\section{I}

From 1872 to 1897, Cantor wrote his basic works devoted to the theory of sets. Russian mathematicians who visited universities of Berlin and Gottingen and read Crelle's Journal, Mathematische Annalen, Acta Mathematica got to know the ideas of the theory of sets. Cantor's ideas gradually permeated research activities and teaching, appeared in press in the form of expositions and translations. We are going to review the history of Cantor's heritage in Russia from 1892 to 1985.

\footnotetext{
* St. Petersburg State University of Architecture and Civil Engineering, St. Petersburg, Russia E-mail: galina.sinkevich@gmail.com
}

Odessa, 1892. I. Y. Timchenko

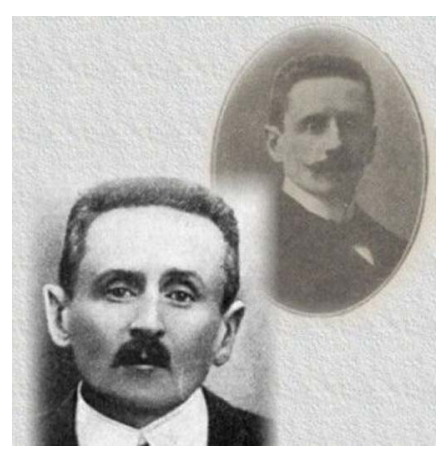

Ivan Y. Timchenko (1863-1939).

We found the first references (1892) to Cantor's works in Russia in works of Ivan Y. Timchenko (1863-1939) who graduated from Novorossiysk University in Odessa in 1885 to subsequently become a professor in Odessa. Timchenko studied astronomy, mathematics and history of mathematics, travelled abroad to work in libraries (in 1890, 1892, 1893, and 1896). Timchenko chose historical analysis of development of the theory of analytical functions as the subject of his MPhil. His work entitled "Basis of the theory of analytical functions" was published in three editions of "Proceedings of the Department of Mathematics of Novorossiysk Scientists" in 1892 and 1899, and presented in 1899 [T1, T2].

His in-depth research covers the period from the ancient world to the late 19th century. In this work, he considered development of basic ideas underlying the theory of analytical functions. The most important of these ideas is the concept of continuity and related concepts of neighbourhood and limit 


\section{The Fate of Russian Translations of Cantor}

\section{by Galina Sinkevich*}

\begin{abstract}
This is the history of translating Cantor's works into Russian from 1892 to 1985 in Odessa, Moscow, Tomsk, Kazan, S.-Petersburg, Leningrad. Mathematicians and philosophers in Russia took the ideas of the theory of sets enthusiastically. Such renowned scholars and scientists as Timchenko, Shatunovsky, Vasiliev, Florensky, Mlodzeevsky, Nekrasov, Zhegalkin, Yushkevich Sr., Fet, Yushkevich Jr., Kolmogorov, and Medvedev took part in their popularisation. In 1970 Academician Pontryagin rated the theory of sets as useless for young mathematicians, and the translated works of Cantor were not published. This article first describes the tragic fate of this translation.
\end{abstract}

MSC 2010 subject classifications: 01A60, 01A70, $01 \mathrm{~A} 85$.

\section{I}

From 1872 to 1897, Cantor wrote his basic works devoted to the theory of sets. Russian mathematicians who visited universities of Berlin and Gottingen and read Crelle's Journal, Mathematische Annalen, Acta Mathematica got to know the ideas of the theory of sets. Cantor's ideas gradually permeated research activities and teaching, appeared in press in the form of expositions and translations. We are going to review the history of Cantor's heritage in Russia from 1892 to 1985.

\footnotetext{
* St. Petersburg State University of Architecture and Civil Engineering, St. Petersburg, Russia E-mail: galina.sinkevich@gmail.com
}

Odessa, 1892. I. Y. Timchenko

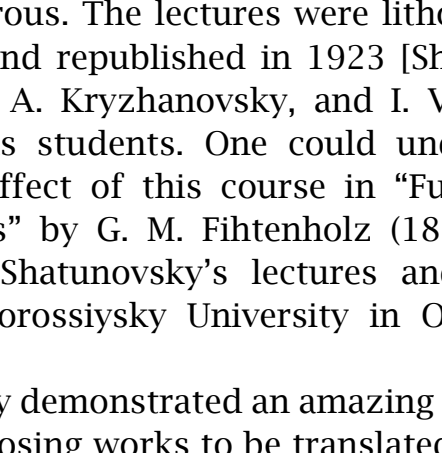

Ivan Y. Timchenko (1863-1939).

We found the first references (1892) to Cantor's works in Russia in works of Ivan Y. Timchenko (1863-1939) who graduated from Novorossiysk University in Odessa in 1885 to subsequently become a professor in Odessa. Timchenko studied astronomy, mathematics and history of mathematics, travelled abroad to work in libraries (in 1890, 1892, 1893, and 1896). Timchenko chose historical analysis of development of the theory of analytical functions as the subject of his MPhil. His work entitled "Basis of the theory of analytical functions" was published in three editions of "Proceedings of the Department of Mathematics of Novorossiysk Scientists" in 1892 and 1899, and presented in 1899 [T1, T2].

His in-depth research covers the period from the ancient world to the late 19th century. In this work, he considered development of basic ideas underlying the theory of analytical functions. The most important of these ideas is the concept of continuity and related concepts of neighbourhood and limit 


\section{The Fate of Russian Translations of Cantor}

\section{by Galina Sinkevich*}

\begin{abstract}
This is the history of translating Cantor's works into Russian from 1892 to 1985 in Odessa, Moscow, Tomsk, Kazan, S.-Petersburg, Leningrad. Mathematicians and philosophers in Russia took the ideas of the theory of sets enthusiastically. Such renowned scholars and scientists as Timchenko, Shatunovsky, Vasiliev, Florensky, Mlodzeevsky, Nekrasov, Zhegalkin, Yushkevich Sr., Fet, Yushkevich Jr., Kolmogorov, and Medvedev took part in their popularisation. In 1970 Academician Pontryagin rated the theory of sets as useless for young mathematicians, and the translated works of Cantor were not published. This article first describes the tragic fate of this translation.
\end{abstract}

MSC 2010 subject classifications: 01A60, 01A70, $01 \mathrm{~A} 85$.

\section{I}

From 1872 to 1897, Cantor wrote his basic works devoted to the theory of sets. Russian mathematicians who visited universities of Berlin and Gottingen and read Crelle's Journal, Mathematische Annalen, Acta Mathematica got to know the ideas of the theory of sets. Cantor's ideas gradually permeated research activities and teaching, appeared in press in the form of expositions and translations. We are going to review the history of Cantor's heritage in Russia from 1892 to 1985.

\footnotetext{
* St. Petersburg State University of Architecture and Civil Engineering, St. Petersburg, Russia E-mail: galina.sinkevich@gmail.com
}

Odessa, 1892. I. Y. Timchenko

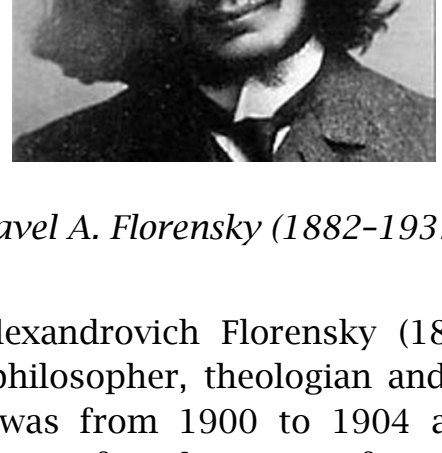

Ivan Y. Timchenko (1863-1939).

We found the first references (1892) to Cantor's works in Russia in works of Ivan Y. Timchenko (1863-1939) who graduated from Novorossiysk University in Odessa in 1885 to subsequently become a professor in Odessa. Timchenko studied astronomy, mathematics and history of mathematics, travelled abroad to work in libraries (in 1890, 1892, 1893, and 1896). Timchenko chose historical analysis of development of the theory of analytical functions as the subject of his MPhil. His work entitled "Basis of the theory of analytical functions" was published in three editions of "Proceedings of the Department of Mathematics of Novorossiysk Scientists" in 1892 and 1899, and presented in 1899 [T1, T2].

His in-depth research covers the period from the ancient world to the late 19th century. In this work, he considered development of basic ideas underlying the theory of analytical functions. The most important of these ideas is the concept of continuity and related concepts of neighbourhood and limit 


\section{The Fate of Russian Translations of Cantor}

\section{by Galina Sinkevich*}

\begin{abstract}
This is the history of translating Cantor's works into Russian from 1892 to 1985 in Odessa, Moscow, Tomsk, Kazan, S.-Petersburg, Leningrad. Mathematicians and philosophers in Russia took the ideas of the theory of sets enthusiastically. Such renowned scholars and scientists as Timchenko, Shatunovsky, Vasiliev, Florensky, Mlodzeevsky, Nekrasov, Zhegalkin, Yushkevich Sr., Fet, Yushkevich Jr., Kolmogorov, and Medvedev took part in their popularisation. In 1970 Academician Pontryagin rated the theory of sets as useless for young mathematicians, and the translated works of Cantor were not published. This article first describes the tragic fate of this translation.
\end{abstract}

MSC 2010 subject classifications: 01A60, 01A70, $01 \mathrm{~A} 85$.

\section{I}

From 1872 to 1897, Cantor wrote his basic works devoted to the theory of sets. Russian mathematicians who visited universities of Berlin and Gottingen and read Crelle's Journal, Mathematische Annalen, Acta Mathematica got to know the ideas of the theory of sets. Cantor's ideas gradually permeated research activities and teaching, appeared in press in the form of expositions and translations. We are going to review the history of Cantor's heritage in Russia from 1892 to 1985.

\footnotetext{
* St. Petersburg State University of Architecture and Civil Engineering, St. Petersburg, Russia E-mail: galina.sinkevich@gmail.com
}

Odessa, 1892. I. Y. Timchenko

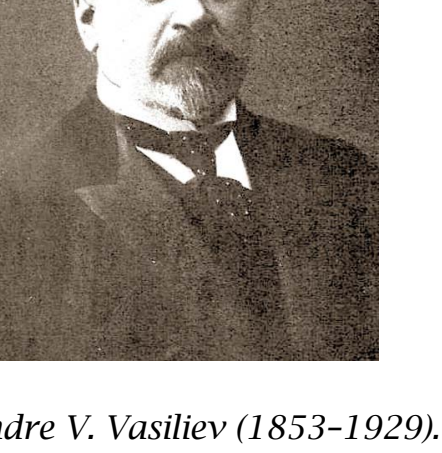

Ivan Y. Timchenko (1863-1939).

We found the first references (1892) to Cantor's works in Russia in works of Ivan Y. Timchenko (1863-1939) who graduated from Novorossiysk University in Odessa in 1885 to subsequently become a professor in Odessa. Timchenko studied astronomy, mathematics and history of mathematics, travelled abroad to work in libraries (in 1890, 1892, 1893, and 1896). Timchenko chose historical analysis of development of the theory of analytical functions as the subject of his MPhil. His work entitled "Basis of the theory of analytical functions" was published in three editions of "Proceedings of the Department of Mathematics of Novorossiysk Scientists" in 1892 and 1899, and presented in 1899 [T1, T2].

His in-depth research covers the period from the ancient world to the late 19th century. In this work, he considered development of basic ideas underlying the theory of analytical functions. The most important of these ideas is the concept of continuity and related concepts of neighbourhood and limit 


\section{The Fate of Russian Translations of Cantor}

\section{by Galina Sinkevich*}

\begin{abstract}
This is the history of translating Cantor's works into Russian from 1892 to 1985 in Odessa, Moscow, Tomsk, Kazan, S.-Petersburg, Leningrad. Mathematicians and philosophers in Russia took the ideas of the theory of sets enthusiastically. Such renowned scholars and scientists as Timchenko, Shatunovsky, Vasiliev, Florensky, Mlodzeevsky, Nekrasov, Zhegalkin, Yushkevich Sr., Fet, Yushkevich Jr., Kolmogorov, and Medvedev took part in their popularisation. In 1970 Academician Pontryagin rated the theory of sets as useless for young mathematicians, and the translated works of Cantor were not published. This article first describes the tragic fate of this translation.
\end{abstract}

MSC 2010 subject classifications: 01A60, 01A70, $01 \mathrm{~A} 85$.

\section{I}

From 1872 to 1897, Cantor wrote his basic works devoted to the theory of sets. Russian mathematicians who visited universities of Berlin and Gottingen and read Crelle's Journal, Mathematische Annalen, Acta Mathematica got to know the ideas of the theory of sets. Cantor's ideas gradually permeated research activities and teaching, appeared in press in the form of expositions and translations. We are going to review the history of Cantor's heritage in Russia from 1892 to 1985.

\footnotetext{
* St. Petersburg State University of Architecture and Civil Engineering, St. Petersburg, Russia E-mail: galina.sinkevich@gmail.com
}

Odessa, 1892. I. Y. Timchenko

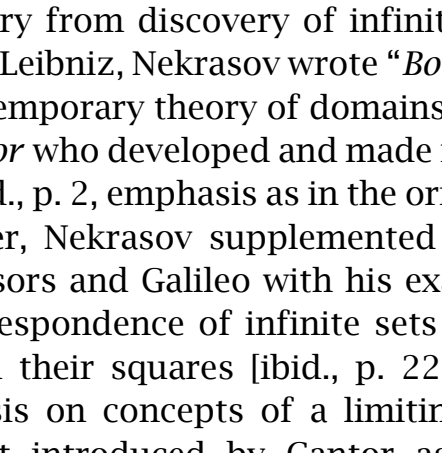

Ivan Y. Timchenko (1863-1939).

We found the first references (1892) to Cantor's works in Russia in works of Ivan Y. Timchenko (1863-1939) who graduated from Novorossiysk University in Odessa in 1885 to subsequently become a professor in Odessa. Timchenko studied astronomy, mathematics and history of mathematics, travelled abroad to work in libraries (in 1890, 1892, 1893, and 1896). Timchenko chose historical analysis of development of the theory of analytical functions as the subject of his MPhil. His work entitled "Basis of the theory of analytical functions" was published in three editions of "Proceedings of the Department of Mathematics of Novorossiysk Scientists" in 1892 and 1899, and presented in 1899 [T1, T2].

His in-depth research covers the period from the ancient world to the late 19th century. In this work, he considered development of basic ideas underlying the theory of analytical functions. The most important of these ideas is the concept of continuity and related concepts of neighbourhood and limit 


\section{The Fate of Russian Translations of Cantor}

\section{by Galina Sinkevich*}

\begin{abstract}
This is the history of translating Cantor's works into Russian from 1892 to 1985 in Odessa, Moscow, Tomsk, Kazan, S.-Petersburg, Leningrad. Mathematicians and philosophers in Russia took the ideas of the theory of sets enthusiastically. Such renowned scholars and scientists as Timchenko, Shatunovsky, Vasiliev, Florensky, Mlodzeevsky, Nekrasov, Zhegalkin, Yushkevich Sr., Fet, Yushkevich Jr., Kolmogorov, and Medvedev took part in their popularisation. In 1970 Academician Pontryagin rated the theory of sets as useless for young mathematicians, and the translated works of Cantor were not published. This article first describes the tragic fate of this translation.
\end{abstract}

MSC 2010 subject classifications: 01A60, 01A70, $01 \mathrm{~A} 85$.

\section{I}

From 1872 to 1897, Cantor wrote his basic works devoted to the theory of sets. Russian mathematicians who visited universities of Berlin and Gottingen and read Crelle's Journal, Mathematische Annalen, Acta Mathematica got to know the ideas of the theory of sets. Cantor's ideas gradually permeated research activities and teaching, appeared in press in the form of expositions and translations. We are going to review the history of Cantor's heritage in Russia from 1892 to 1985.

\footnotetext{
* St. Petersburg State University of Architecture and Civil Engineering, St. Petersburg, Russia E-mail: galina.sinkevich@gmail.com
}

Odessa, 1892. I. Y. Timchenko

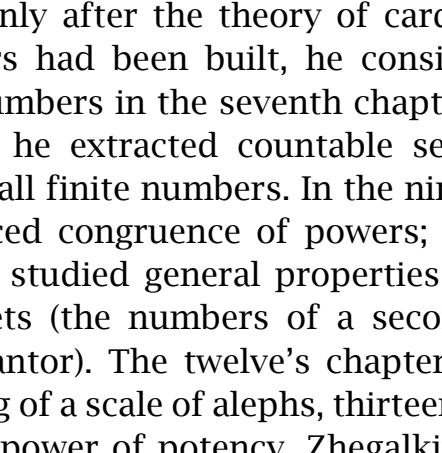

Ivan Y. Timchenko (1863-1939).

We found the first references (1892) to Cantor's works in Russia in works of Ivan Y. Timchenko (1863-1939) who graduated from Novorossiysk University in Odessa in 1885 to subsequently become a professor in Odessa. Timchenko studied astronomy, mathematics and history of mathematics, travelled abroad to work in libraries (in 1890, 1892, 1893, and 1896). Timchenko chose historical analysis of development of the theory of analytical functions as the subject of his MPhil. His work entitled "Basis of the theory of analytical functions" was published in three editions of "Proceedings of the Department of Mathematics of Novorossiysk Scientists" in 1892 and 1899, and presented in 1899 [T1, T2].

His in-depth research covers the period from the ancient world to the late 19th century. In this work, he considered development of basic ideas underlying the theory of analytical functions. The most important of these ideas is the concept of continuity and related concepts of neighbourhood and limit 


\section{The Fate of Russian Translations of Cantor}

\section{by Galina Sinkevich*}

\begin{abstract}
This is the history of translating Cantor's works into Russian from 1892 to 1985 in Odessa, Moscow, Tomsk, Kazan, S.-Petersburg, Leningrad. Mathematicians and philosophers in Russia took the ideas of the theory of sets enthusiastically. Such renowned scholars and scientists as Timchenko, Shatunovsky, Vasiliev, Florensky, Mlodzeevsky, Nekrasov, Zhegalkin, Yushkevich Sr., Fet, Yushkevich Jr., Kolmogorov, and Medvedev took part in their popularisation. In 1970 Academician Pontryagin rated the theory of sets as useless for young mathematicians, and the translated works of Cantor were not published. This article first describes the tragic fate of this translation.
\end{abstract}

MSC 2010 subject classifications: 01A60, 01A70, $01 \mathrm{~A} 85$.

\section{I}

From 1872 to 1897, Cantor wrote his basic works devoted to the theory of sets. Russian mathematicians who visited universities of Berlin and Gottingen and read Crelle's Journal, Mathematische Annalen, Acta Mathematica got to know the ideas of the theory of sets. Cantor's ideas gradually permeated research activities and teaching, appeared in press in the form of expositions and translations. We are going to review the history of Cantor's heritage in Russia from 1892 to 1985.

\footnotetext{
* St. Petersburg State University of Architecture and Civil Engineering, St. Petersburg, Russia E-mail: galina.sinkevich@gmail.com
}

Odessa, 1892. I. Y. Timchenko

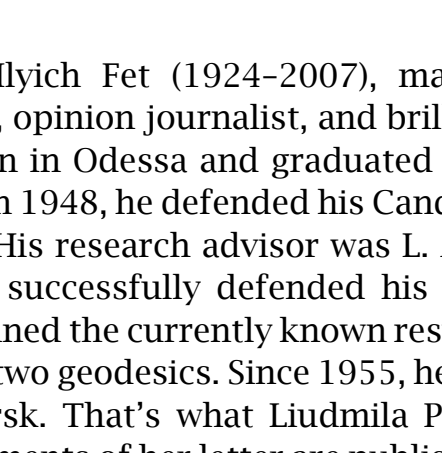

Ivan Y. Timchenko (1863-1939).

We found the first references (1892) to Cantor's works in Russia in works of Ivan Y. Timchenko (1863-1939) who graduated from Novorossiysk University in Odessa in 1885 to subsequently become a professor in Odessa. Timchenko studied astronomy, mathematics and history of mathematics, travelled abroad to work in libraries (in 1890, 1892, 1893, and 1896). Timchenko chose historical analysis of development of the theory of analytical functions as the subject of his MPhil. His work entitled "Basis of the theory of analytical functions" was published in three editions of "Proceedings of the Department of Mathematics of Novorossiysk Scientists" in 1892 and 1899, and presented in 1899 [T1, T2].

His in-depth research covers the period from the ancient world to the late 19th century. In this work, he considered development of basic ideas underlying the theory of analytical functions. The most important of these ideas is the concept of continuity and related concepts of neighbourhood and limit 


\section{The Fate of Russian Translations of Cantor}

\section{by Galina Sinkevich*}

\begin{abstract}
This is the history of translating Cantor's works into Russian from 1892 to 1985 in Odessa, Moscow, Tomsk, Kazan, S.-Petersburg, Leningrad. Mathematicians and philosophers in Russia took the ideas of the theory of sets enthusiastically. Such renowned scholars and scientists as Timchenko, Shatunovsky, Vasiliev, Florensky, Mlodzeevsky, Nekrasov, Zhegalkin, Yushkevich Sr., Fet, Yushkevich Jr., Kolmogorov, and Medvedev took part in their popularisation. In 1970 Academician Pontryagin rated the theory of sets as useless for young mathematicians, and the translated works of Cantor were not published. This article first describes the tragic fate of this translation.
\end{abstract}

MSC 2010 subject classifications: 01A60, 01A70, $01 \mathrm{~A} 85$.

\section{I}

From 1872 to 1897, Cantor wrote his basic works devoted to the theory of sets. Russian mathematicians who visited universities of Berlin and Gottingen and read Crelle's Journal, Mathematische Annalen, Acta Mathematica got to know the ideas of the theory of sets. Cantor's ideas gradually permeated research activities and teaching, appeared in press in the form of expositions and translations. We are going to review the history of Cantor's heritage in Russia from 1892 to 1985.

\footnotetext{
* St. Petersburg State University of Architecture and Civil Engineering, St. Petersburg, Russia E-mail: galina.sinkevich@gmail.com
}

Odessa, 1892. I. Y. Timchenko

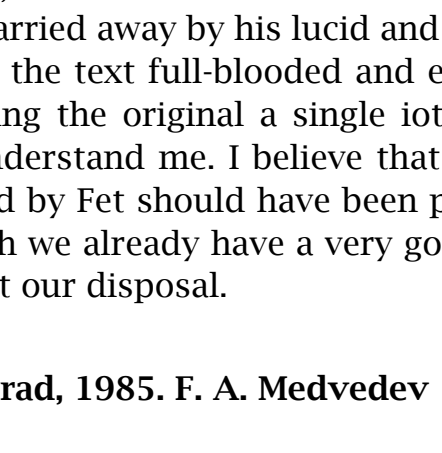

Ivan Y. Timchenko (1863-1939).

We found the first references (1892) to Cantor's works in Russia in works of Ivan Y. Timchenko (1863-1939) who graduated from Novorossiysk University in Odessa in 1885 to subsequently become a professor in Odessa. Timchenko studied astronomy, mathematics and history of mathematics, travelled abroad to work in libraries (in 1890, 1892, 1893, and 1896). Timchenko chose historical analysis of development of the theory of analytical functions as the subject of his MPhil. His work entitled "Basis of the theory of analytical functions" was published in three editions of "Proceedings of the Department of Mathematics of Novorossiysk Scientists" in 1892 and 1899, and presented in 1899 [T1, T2].

His in-depth research covers the period from the ancient world to the late 19th century. In this work, he considered development of basic ideas underlying the theory of analytical functions. The most important of these ideas is the concept of continuity and related concepts of neighbourhood and limit 


\section{The Fate of Russian Translations of Cantor}

\section{by Galina Sinkevich*}

\begin{abstract}
This is the history of translating Cantor's works into Russian from 1892 to 1985 in Odessa, Moscow, Tomsk, Kazan, S.-Petersburg, Leningrad. Mathematicians and philosophers in Russia took the ideas of the theory of sets enthusiastically. Such renowned scholars and scientists as Timchenko, Shatunovsky, Vasiliev, Florensky, Mlodzeevsky, Nekrasov, Zhegalkin, Yushkevich Sr., Fet, Yushkevich Jr., Kolmogorov, and Medvedev took part in their popularisation. In 1970 Academician Pontryagin rated the theory of sets as useless for young mathematicians, and the translated works of Cantor were not published. This article first describes the tragic fate of this translation.
\end{abstract}

MSC 2010 subject classifications: 01A60, 01A70, $01 \mathrm{~A} 85$.

\section{I}

From 1872 to 1897, Cantor wrote his basic works devoted to the theory of sets. Russian mathematicians who visited universities of Berlin and Gottingen and read Crelle's Journal, Mathematische Annalen, Acta Mathematica got to know the ideas of the theory of sets. Cantor's ideas gradually permeated research activities and teaching, appeared in press in the form of expositions and translations. We are going to review the history of Cantor's heritage in Russia from 1892 to 1985.

\footnotetext{
* St. Petersburg State University of Architecture and Civil Engineering, St. Petersburg, Russia E-mail: galina.sinkevich@gmail.com
}

Odessa, 1892. I. Y. Timchenko

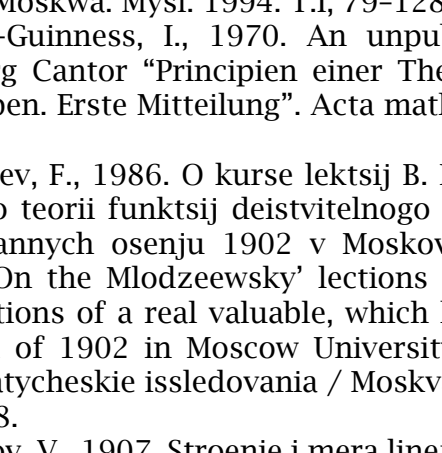

Ivan Y. Timchenko (1863-1939).

We found the first references (1892) to Cantor's works in Russia in works of Ivan Y. Timchenko (1863-1939) who graduated from Novorossiysk University in Odessa in 1885 to subsequently become a professor in Odessa. Timchenko studied astronomy, mathematics and history of mathematics, travelled abroad to work in libraries (in 1890, 1892, 1893, and 1896). Timchenko chose historical analysis of development of the theory of analytical functions as the subject of his MPhil. His work entitled "Basis of the theory of analytical functions" was published in three editions of "Proceedings of the Department of Mathematics of Novorossiysk Scientists" in 1892 and 1899, and presented in 1899 [T1, T2].

His in-depth research covers the period from the ancient world to the late 19th century. In this work, he considered development of basic ideas underlying the theory of analytical functions. The most important of these ideas is the concept of continuity and related concepts of neighbourhood and limit 


\section{The Fate of Russian Translations of Cantor}

\section{by Galina Sinkevich*}

\begin{abstract}
This is the history of translating Cantor's works into Russian from 1892 to 1985 in Odessa, Moscow, Tomsk, Kazan, S.-Petersburg, Leningrad. Mathematicians and philosophers in Russia took the ideas of the theory of sets enthusiastically. Such renowned scholars and scientists as Timchenko, Shatunovsky, Vasiliev, Florensky, Mlodzeevsky, Nekrasov, Zhegalkin, Yushkevich Sr., Fet, Yushkevich Jr., Kolmogorov, and Medvedev took part in their popularisation. In 1970 Academician Pontryagin rated the theory of sets as useless for young mathematicians, and the translated works of Cantor were not published. This article first describes the tragic fate of this translation.
\end{abstract}

MSC 2010 subject classifications: 01A60, 01A70, $01 \mathrm{~A} 85$.

\section{I}

From 1872 to 1897, Cantor wrote his basic works devoted to the theory of sets. Russian mathematicians who visited universities of Berlin and Gottingen and read Crelle's Journal, Mathematische Annalen, Acta Mathematica got to know the ideas of the theory of sets. Cantor's ideas gradually permeated research activities and teaching, appeared in press in the form of expositions and translations. We are going to review the history of Cantor's heritage in Russia from 1892 to 1985.

\footnotetext{
* St. Petersburg State University of Architecture and Civil Engineering, St. Petersburg, Russia E-mail: galina.sinkevich@gmail.com
}

Odessa, 1892. I. Y. Timchenko

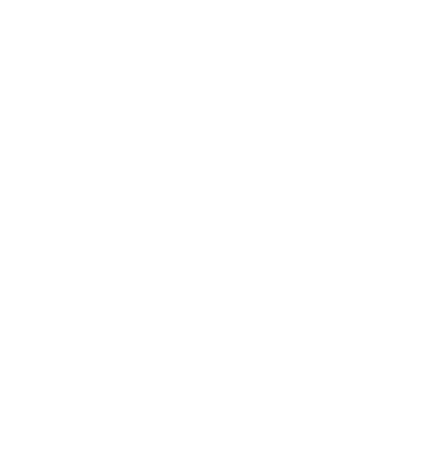

Ivan Y. Timchenko (1863-1939).

We found the first references (1892) to Cantor's works in Russia in works of Ivan Y. Timchenko (1863-1939) who graduated from Novorossiysk University in Odessa in 1885 to subsequently become a professor in Odessa. Timchenko studied astronomy, mathematics and history of mathematics, travelled abroad to work in libraries (in 1890, 1892, 1893, and 1896). Timchenko chose historical analysis of development of the theory of analytical functions as the subject of his MPhil. His work entitled "Basis of the theory of analytical functions" was published in three editions of "Proceedings of the Department of Mathematics of Novorossiysk Scientists" in 1892 and 1899, and presented in 1899 [T1, T2].

His in-depth research covers the period from the ancient world to the late 19th century. In this work, he considered development of basic ideas underlying the theory of analytical functions. The most important of these ideas is the concept of continuity and related concepts of neighbourhood and limit 